\title{
Articles
}

\section{Time is Double the Trouble: Zeno's Moving Rows}

\author{
Barbara Sattler
}

Imagine a little provincial train station south of Elea, which has its platform divided into four equal sections, 1, 2, 3, 4. In fact it is so provincial that its sole platform is only as long as the two major trains that pass through this station every morning (they do not stop there any longer). These two trains move in opposite directions, one going south, the Southern Express, the other going north, the Northern Arrow. Both trains consist of one locomotive and three carriages, all of equal length; and, as it happens, each carriage is of the same length as a section of the platform. Since the two trains normally arrive at the same time, they also pass each other in the station. And they travel with the same speed, traversing one section in one second. Accordingly, if we look at the Southern Express and the Northern Arrow at the moment when they are right in the middle of the platform, that is, right before they start passing each other, we can say that it will take the Southern Express two seconds to pass the remaining two sections of the train station. Yet a person sitting in the Northern Arrow looking at the Southern Express will see that his train passes the four sections of the Southern Express during the same two seconds, so that the two trains will be aligned at the end of the two seconds. But since one section was supposed to be passed in one second, it seems that double the time (needed to traverse the 4 sections of the Southern Express, which the Northern Arrow has passed) equals half the time (needed to pass the 2 sections of the train station the Southern Express has passed). ${ }^{1}$

This scenario is roughly what Zeno employs in his so-called 'Moving Rows Paradox',2 a paradox that is handed down to us in Aristotle's Physics. For the secondary literature, this paradox is somewhat of a stepchild among Zeno's paradoxes of motion. It is often simply omitted in accounts of the four paradoxes, while the other children of this group have enjoyed widespread popularity among philosophers as paradigms of well set up and fruitful paradoxes. And the text of the manuscripts is often considered to be simply a mess (e.g., Wagner in Aristoteles 1995,639$)$. Roughly speaking, there have been three main reactions to

1 The idea of translating Zeno's stadium into a train station setting was originally introduced in a more limited version by Sorabji 1983. For the set-up of an ancient stadium cf. below.

2 Some scholars refer to this fourth paradox of motion as the 'Stadium paradox', a title that has, however, also been used in order to refer to the first paradox of motion, viz., the paradox that in a finite time a runner will never be able to reach the end of a finite race course (or cannot even get started). I will use the title 'Moving Rows' for the fourth paradox of motion here in order to avoid any confusion. 
this paradox in the scholarly literature: (a) one group thinks of it as weak and uninteresting, since it presents no real paradox at all; (b) a second group considers it to be based on an oversight regarding some kind of relativity involved in motion, though an understandable one; while (c) a third group thinks we can find something of genuine philosophical interest in it, if the paradox is understood as directed against a rather limited target, namely, against atomism.

I aim to show that none of the secondary literature so far has given the right diagnosis of the paradox; and that once we see what is really the problem the paradox alerts us to, we can also value it as a generally interesting paradox: as a paradox that is neither a mere oversight on Zeno's part, nor directed solely against a limited (and perhaps, at Zeno's time, not even existent) group of philosophers. With this new analysis in place, we can see that the paradox raises a significant and by no means trivial problem concerning the relationship between time, space, and motion. My new diagnosis will also make it clear why Zeno has to employ such elaborate machinery in order to bring out the point this paradox aims to make, machinery that has led to quite some puzzlement among Zeno's readers (and to confusion among the scribes).

So I attempt to give $(\alpha)$ a new diagnosis of the paradox, which $(\beta)$ should lead us to a new evaluation of it. $(\alpha)$ I argue that the paradox rests on the assumption that the time of a motion depends directly on the distance covered, in the sense that the distance covered is the only relevant factor for determining the time taken. While this assumption is false, it is in the immediate neighbourhood of a true one - that the time of a motion is correlated with the distance covered, so that the distance covered is one, but only one factor for determining the time. What makes it difficult to see that this is the problematic assumption on which the paradox rests is that, first, for many situations the false assumption, precisely because it is in the neighbourhood of a true one, gets the correct results; and, second, that it is difficult to formulate precisely how time and space are correlated.

As for the first difficulty, let me give an example that shows that there are ordinary situations where we do indeed rely on the distance covered as the only factor determining the time required for a motion. Let us assume you live 20 kilometres from your workplace and it usually takes you 20 minutes to get there. Now you tell me you are thinking of moving to another house that will be 40 kilometres away from your workplace. Chances are that I then conclude that it will now take you 40 minutes to work, so that I am actually relying on the distance as the only factor determining the time it takes you to work. Suppose you tell me, however, that if you move it will still take you 20 minutes to get to work (unbeknownst to me, there is a motorway close to the new house, so that you will be able to cover the 40 kilometres in the same 20 minutes). I may be puzzled at first that what seemed to me double the time turns out to be half the time, that is, half the time I expected it would take you to get to work. We see that the false assumption is a plausible default assumption on which we routinely rely, espe- 
cially when other information is lacking. ${ }^{3}$ Zeno's paradox very clearly spells out the problematic consequences reliance on this false assumption can have in certain situations.

As for the second difficulty, that it is difficult to formulate precisely how time and space are correlated, Aristotle's treatment of the paradox will be evidence that even if it seems clear that something is wrong with the account given in Zeno's paradox, it is not easy to say what would be the right account to give instead -we shall see that this requires an understanding of motion as a certain correlation of time and space that we do not find in ancient times. So Zeno's paradox is exploiting not only that we often rely on the false assumption, but also that is not easy to spell out the right one.

$(\beta)$ The exact evaluation resulting from this analysis depends on what we think the criteria for a good paradox to be. There are several possible criteria we may employ: we may claim a paradox is good only if no solution seems possible, or if no solution so far has been found. Or we may think it is enough for a good paradox in case it is convincing that people employ the (apparently plausible but in fact problematic) assumptions it relies on and a solution is not simply obvious, irrespective of whether we have an agreed upon resolution. I would count the Moving Rows paradox as a good paradox in the last sense (though we do not have an agreed upon resolution for this paradox so far, since we do not have an agreed upon diagnosis). But in any case I hope my reconstruction clearly shows that it is an interesting paradox, in the sense in which we take the other paradoxes of motion to be interesting paradoxes, without this necessarily meaning that we fall prey to it. It can be understood as a kind of challenge to formulate the true assumption that the false assumption is in the neighbourhood of.

The problem raised by this paradox can be demonstrated to connect it with the first two of Zeno's motion paradoxes. These two paradoxes not only bring up the problem how a finite distance can contain infinitely many parts, but also how the infinitely many spatial parts can be covered in a finite time, and thus how the time and the space of a motion are related. Together the four paradoxes of motion raise many of the most important problems with conceptualizing motion in ancient times, problems that Aristotle attempts to solve in his Physics. ${ }^{4}$ However, here I will have to leave out the connection of the Moving Rows paradox to the other paradoxes. I will begin with a quick textual reconstruction of the paradox of the Moving Rows before sketching the main philosophical problem the paradox raises on my interpretation. I will finish with demonstrating that none of the three kinds of interpretations on offer in the secondary literature so far provides us with an adequate account of the paradox.

\footnotetext{
${ }^{3}$ Information, that is, on varying or constant speed, on uniformity or non-uniformity of motion. However, when pressed we may not assert that distance is the only factor relevant to determining the time taken.

${ }^{4}$ The aim of Zeno's paradoxes seems to me to be, as Plato first suggested, to support the position of Parmenides. However, this is a point that is independent of the specific interpretation of Zeno's paradox I am presenting, and thus I will not go into it here.
} 


\section{Reconstruction of Zeno's Paradox}

There is one respect in which the the bad press concerning this paradox is absolutely right - the text is indeed a big mess; prima facie it seems even beyond recovery. While I think it is indeed possible to produce a defensible text, doing so is a different project, and I have tried to do this elsewhere. I will therefore pass over most of the philological difficulties here. For our present purposes we will follow manuscripts $\mathrm{H}$ and $\mathrm{F}$ for the most part. ${ }^{5}$ In addition, we will build on the fact that most people agree on the starting and end position. And we will assume, employing a principle of charity, that the paradoxical result-double the time equals half the time-has to be derived from the starting and end position following plausible and thus seemingly correct reasoning. With this in mind, consider Zeno's paradox as handed down to us in Aristotle's Physics 239b33-240a17:

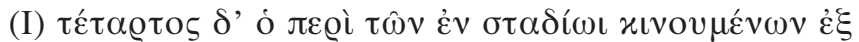

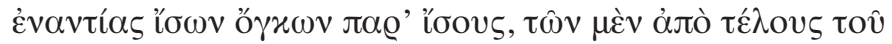

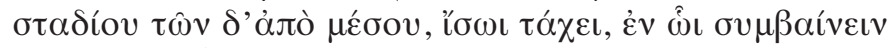

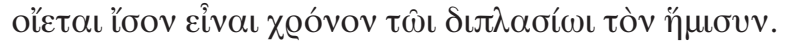

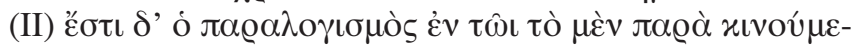

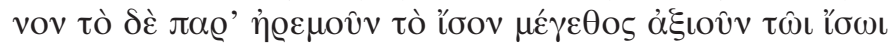

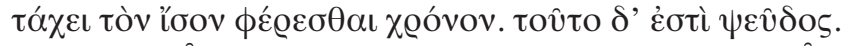

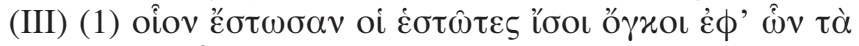

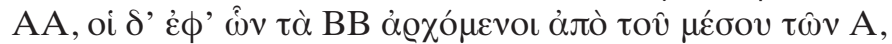

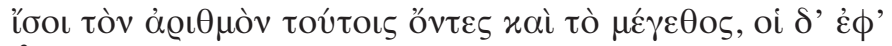

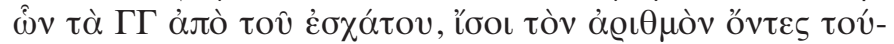

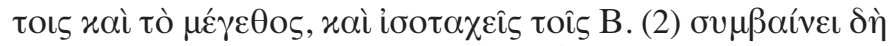

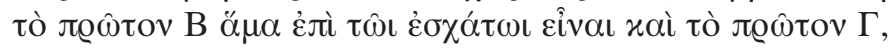

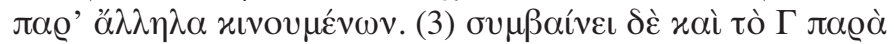

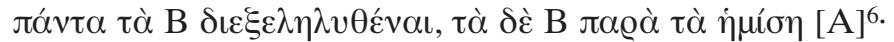

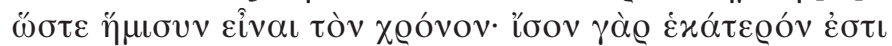

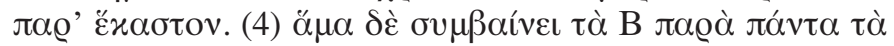

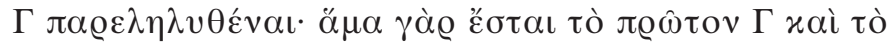

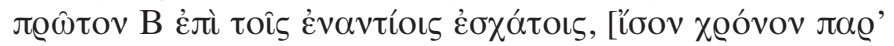

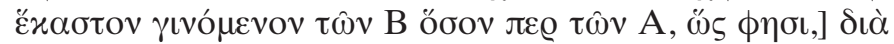

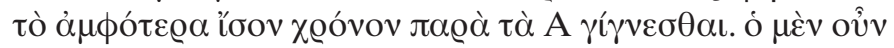

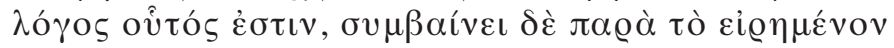
$\psi \varepsilon \hat{v} \delta$ os.

(I) The fourth (argument) is that concerning equal bodies which move alongside equal bodies in the stadium from opposite directions - the ones (starting) from the end of the stadium, the others from the middle-at equal speeds; from this he

\footnotetext{
${ }^{5}$ In Sattler under review I give an explanation of this policy and a defence of those aspects of my reading where (in accordance with Simplicius) it has to deviate from $\mathrm{H}$ and $\mathrm{F}$.

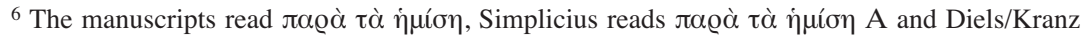
follow him.
} 
thinks it follows that half the time is equal to its double.

(II) The fallacy consists in assuming that a body travelling at an equal speed travels for an equal time past a moving body and a body of the same size at rest. That is false.

(III) (1) For instance let the stationary equal bodies be AA; let $\mathrm{BB}$ be those starting from the middle of the As, equal in number and in magnitude to them; and let $\mathrm{CC}$ be those starting from the end, equal in number and magnitude to them, and equal in speed to the Bs. (2) Now it follows that the first B and the first $\mathrm{C}$ are at the end at the same time, as they are moving past one another. (3) It turns out that the (first) $\mathrm{C}$ has gone past all the Bs, but B (only) past half [the As]; so that the time should be only half. [The next sentence of the text is uncertain between (a) 'For each of them is equal with respect to each' and (b) 'For each of them is for the same time alongside each']. ${ }^{7}$ (4) And at the same time it follows that the first B has passed all the Cs. For at the same time the first B and the first $\mathrm{C}$ will be at opposite ends [it takes an equal time (passing) each of the Bs as each of the As, as he says], because both are an equal time alongside the As. That is the argument, and it rests on the stated falsity (translation by Hardie and Gaye as revised by Barnes, with alterations).

It is useful to divide Aristotle's account of this paradox into three main parts, indicated with the help of Roman numerals in the text above. The third part in turn is divided into four steps, indicated with the help of Arabic numerals. ${ }^{8}$ Let us start with the three main parts: (I) Firstly, Aristotle very briefly sketches the setup of the paradox and names the paradoxical result - half the time equals double the time. (II) In the second part Aristotle determines the fallacious conclusion on which according to him the paradox rests, namely, on the assumption that if something moves with constant speed, it will need the same time to pass something moving as to pass something resting. However, he does not give a diagnosis in the sense that he would explain how we could reasonably get to the fallacious conclusion. (III) Finally, Aristotle fills out the initial sketch of the paradox with the help of an example and then rounds off the account with a back reference to his analysis of the paradox. In order to reconstruct Zeno's paradox, we have to consider how the example given in this third part leads to the paradox named in the first part. ${ }^{9}$

${ }^{7}$ I discuss the alternatives below.

${ }^{8}$ For the division of the text, cf. also Barnes 1982, 286, who delimits the first two parts of the paradox in the same way as I do, but then continues with ten further parts, which I will treat as four subdivisions of the third part.

${ }^{9}$ Ferber 1995, 22-23 and Mansfeld 1982 think that this third part is either completely (so Ferber) or in part (so Mansfeld) by Aristotle, not by Zeno. However, Ferber's reconstruction is in general rather far away from the text, cf. Knorr's 1983 review of it. And Mansfeld, in order to get a paradox at 
Following Aristotle's text, we find four steps in this third part: The first step explains the starting position of the bodies in the stadium, the second one provides the end position, and steps three and four give the conceptual description of the rows' movement that leads into the paradox. Going through these four steps of the third main part in detail will allow us to reconstruct the philosophical problem on which the paradox turns.

(1) We have three different rows of bodies in a stadium, As, Bs, Cs, all of the same number and size. ${ }^{10}$ In the beginning the Bs are in the middle of the stadium as well as in the middle of the As; the Cs are at the end of the stadium and at the end of the Bs. ${ }^{11}$ So we can represent the starting position as follows: ${ }^{12}$

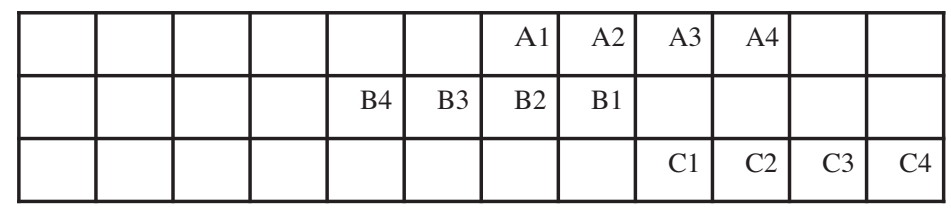

all, has to assume, against the explicit claim of the text even in the part he thinks is genuinely Zenonian, that one of the rows is moving twice as fast as the other (cf., e.g., pp. 6 and 16). I do not deny that there may be some Aristotelian language used in this example, for instance, as Ferber 1995, 23 and 113 claims, the use of letter symbols; but without this third part there is no paradox to be derived from the text as handed down to us. And while Aristotle sometime presents the ideas and views of his predecessors from his particular perspective, which we may not always think is adequate to these earlier thinkers, I do not think we have any good reason to assume that Aristotle would simply make up such an extended piece of reasoning and sell it as the idea of one of his predecessors (I have argued elsewhere against Vlastos' assumption that Aristotle employs his own technical notion of the now when giving an account of the arrow paradox, which is Mansfeld's alleged example-the only one he gives - of such a practise in Aristotle). On the contrary, Aristotle is quite clear in marking off Zeno's idea (part one and most of part three) from his own evaluation (part two and the last sentence of part three).

${ }^{10}$ The first main part (I) solely seems to mention two rows, both of which are moving. The third, resting row is introduced only in the example here in the third main part (III). While the starting position of the two moving rows is determined with the help of the stadium in the first part, the third part seems to determine it in respect to the As. It is not spelt out in the text how the first situation translates into the second, which seems to have led to some confusion in the manuscripts concerning the starting position. Accordingly, several scholars have removed the As from the scene altogether, e.g., Mansfeld 1982, Ferber 1995, and Wicksteed and Cornford in Aristotle 1934, following an unpublished manuscript of Ross. I agree with these scholars that the third row is actually not necessary for a reconstruction of the argument-the resting stadium would suffice as a point of reference. However, we will see below that we nevertheless need something resting that is of the same size as the moving rows in respect to which the motion of one of the moving rows is considered in order to create Zeno's paradox; but this could also be units marked out in the stadium that are of the same size as the bodies of the moving rows. Those scholars who try to reconstruct the starting and end position without such resting units, as e.g., Mansfeld and Ferber, have to introduce additional assumptions not mentioned in the text or even going against what is explicitly said in the text in order to be able to derive a paradox.

${ }^{11}$ Although the Bs start from the middle of the As in one direction as do the Cs in the other direction, the text does not state it in this way. This is because the Bs are characterized with respect to the As, while the Cs are characterized solely with respect to the Bs.

${ }^{12}$ In the manuscripts we find everything from one to four As; but we will see that the exact number of bodies in each row is not essential for understanding the paradox; it is only essential that there 
Given an ancient stadium, which had roughly a paperclip form and can have turning points on either side, we have to imagine either a diaulos-race (a doublestadion race), which was run out and back once around the far turning-post, or a dolichos-race of several laps - in any case a race that involved running around a turning point, since this explains why different onkoi are moving in different directions.${ }^{13}$ So for the starting position of the paradox, Zeno has captured not the actual starting point of an ancient race, but a particular situation in which one row, let us assume the Cs in our drawing, have already passed the relevant turning point we suppose to be on the right side, and are hence moving away from it to the left, while the Bs are travelling towards it and thus move to the right. An ancient race would not have stationary bodies standing in the race-course, but we can assume the As to have a breakdown, and thus to be resting. ${ }^{14}$

(2) The As are at rest while the Bs and Cs move in opposite directions past each other. Thus the first B arrives at the end of the As at the same time as the first $\mathrm{C}$ does. We can represent this end position as follows:

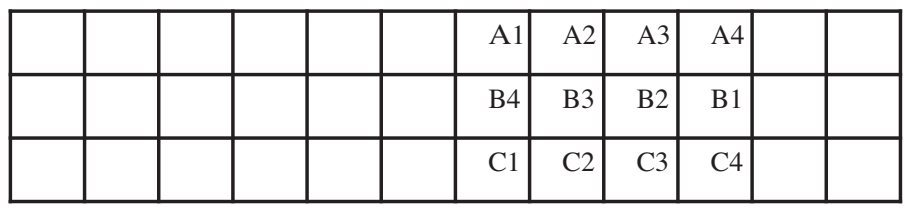

It is the subsequent account of this state of affairs that creates a paradox. The text shows this in two steps, which I have labelled 3 and 4 respectively:

(3) We can understand the third step as a conclusion with three premises, one of which is stated only after the actual conclusion is given:

(1) C has passed all the Bs (Premise)

(2) B has passed only half the As (Premise) $)^{15}$

(3) Thus the time [which B needs for its movement past the As] is only half [the time $\mathrm{C}$ needs to move past the Bs] (from 1, 2 , and a missing premise).

Depending on the reading of lines 12-13 - where I gave two alternatives abovethe third premise is either:

For each of them [either those passing, i.e., the Bs and Cs, or those passed, i.e., the Bs and As; and either the whole row or

is the same number in each row. I have chosen four letters for the simple reason that four letters give a clear graph and have been frequently used in the tradition commenting on this paradox; cf. also Wagner in Aristoteles 1995, 640.

${ }^{13}$ For the set up of these ancient races see, e.g., Miller 2004, 31-35 and 44-45. Ross 1936, 663 tried to argue that what we understand as the middle of the stadium is actually the middle of the race, and thus the turning point at one end of the stadium. However, Ross himself admitted that there is no evidence that the Greek word for the middle, to meson, was ever used to indicate such a middle qua a turning point, cf. also Mansfeld 1982, 5 and Ferber 1995, 24.

${ }^{14}$ The stationary bodies seem to be introduced as merely hypothetical ("' $\left.\sigma \tau \omega \sigma \alpha v\right)$.

${ }^{15}$ For proof that this is how to understand the text of the paradox, cf. Sattler under review. 
their individual bodies] is equal in comparison with each [i.e., either the As, Bs, and Cs, or each of the individual bodies in all three of the sets]. Or:

For each of them is for the same time (add chronon) alongside each.

On the second reading the reason why they are alongside each for the same time is that 'it takes the same time (passing) each of the Bs as each of the As, so he says' (lines 240a15-16), since it is 'equal bodies which move alongside equal bodies' (lines 239b34), i.e., the As, Bs, and Cs are all of equal extension.

Whichever of the two translations one prefers, for the conclusion the crucial assumption in both is that each element (either the individual body represented by a letter or the whole row) is of the same size or extension, so it seems to take the same time to pass any of the bodies and any part of the row. Accordingly, it should take twice as much time to pass 4 Bs as it takes to pass 2 As. The apparent basis of this premise was mentioned already at the very beginning of the whole passage in part (I), namely, that 'equal bodies move alongside equal bodies'. Although the implications of this equality were not spelt out when it was first introduced, it is unproblematic to refer to this premise and its implications only after the conclusion.

$\mathrm{Up}$ to this point it seems that to pass all the Bs it took $\mathrm{C}$ double the time that it took $\mathrm{B}$ to pass half the As, for $\mathrm{C}$ has passed twice as many Bs as B has passed As. Given the fact that the As are resting while the Bs are moving, the problem already implied is made explicit in the following step.

(4) While the first B has passed half the As, it has also passed all the Cs. This follows from the characterization of the end position in step 2, that the first B is at the end at the same time as the first $\mathrm{C}$. And this simultaneous arrival in turn follows from the fact that 'both are an equal time alongside the As', i.e., B and C have passed the same number of As with - as stated above - the same speed. With the starting position we have assumed, the first $\mathrm{B}$ and the first $\mathrm{C}$ will be at opposite ends of the A row simultaneously. So, all Zeno is doing in step four is actually cashing out the implications of the initial set up of the paradox:

(1) The first B and the first $\mathrm{C}$ each start in the middle of the As (Premise)

(2) The Bs and Cs move with the same speed (Premise)

(3) The Bs and Cs are alongside the As for the same time (from $1,2)$

(4) The first B is at the end (of the As) at the same time as the first $\mathrm{C}$ (from 1, 3)

(5) While the first B has passed half the As, it has also passed all the Cs (from 1,4)

Step three and four in Aristotle's text together fully unfold the paradox: the first $\mathrm{B}$ seems to have needed only half the time for its movement past the As that the first $\mathrm{C}$ needed to pass the Bs, for B covered only half as many As as C covered Bs. Simultaneously, it appears that it took B double the time of this half time 
since it passed twice as many Cs as As. ${ }^{16}$ Thus, we arrive at the paradox stated at the beginning that 'half the time is equal to its double', $\mathrm{t} / 2=\mathrm{t}$. B is simultaneously engaged in an enterprise that is meant to take half the time it takes $\mathrm{C}$ for its enterprise (viz., passing half the number of bodies; B passes two As), as well as in an enterprise that takes the same time as C's enterprise (passing as many bodies as $\mathrm{C}$ does; B passes four Cs); the former is described in step 3, the latter in step $4 .{ }^{17}$

\section{The Philosophical Problem}

Zeno's elaborate account of rows moving in a stadium leads to the paradox that row B seems to have been engaged at the same time in an enterprise taking two units of time and in an enterprise taking four units of time. The logical foundation on which this paradox rests is that Zeno implicitly employs a seemingly plausible and unproblematic assumption, namely, that the time of a motion is dependent solely on the space traversed. This assumption is not made explicit anywhere in the paradox, but, as I want to show in the following, it is a necessary implication of the fact that the time of a motion is inferred directly from the distance covered.

Let us look at time's dependence on space more closely. By a 'dependent magnitude' I simply mean here a magnitude whose value can be immediately derived from the magnitude it is dependent on. For example, in the function $y=x^{2}$, the value of $y$ can be immediately derived from $x$. Likewise, I argue, in the paradox time is treated as a magnitude whose value (i.e., the amount of time units needed for the movement) can be immediately derived from the value of space (i.e., the amount of space units covered by the motion). Time is treated as if it were a mere function of space. ${ }^{18}$ This dependence relation is an asymmetrical relation in which space is the only relevant factor in determining time.${ }^{19}$ Hence, the amount of time required is determined solely by the amount of distance travelled, i.e.,

${ }^{16}$ The same problem arises with the Cs of course.

17 'Half the time is equal to its double' in Greek could either mean $t / 2=2 t$ or $t / 2=t$. Gaye 1910, 100ff. argues for the former as the more dramatic one, 'Zeno would have considered that, the more extravagant the paradox, the greater would be the cogency of the argument'. However, we saw in the reconstruction above that the latter interpretation is the one the logic of the argument employs - the time required to pass two letter elements is equal to the time needed to pass four elements, $t / 2=t$. Understanding the double as the normal correlative of the half (so that $\mathrm{t}$ would be understood as the double of the half $\mathrm{t} / 2$ ) is widespread in Aristotle's work; cf., for instance, his Categories. There we are told that relatives are spoken of in relation to their correlatives and one example given for this is

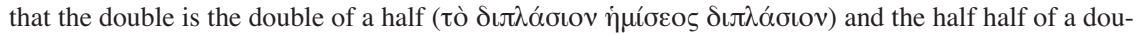

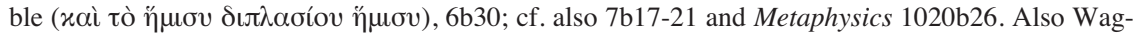
ner in Aristoteles 1995, 640 argues that this latter interpretation fits the Greek, and especially Aristotle's Greek, very naturally.

${ }^{18}$ It should be noted that in this paradox the space covered is expressed as the amount of bodies passed-e.g., we could imagine one body being $40 \mathrm{~cm}$ long, so covering two bodies would amount to covering $80 \mathrm{~cm}$.

${ }^{19} \mathrm{As}$, in the example given, the function is asymmetrical, since the value of $\mathrm{x}$ cannot be immediately and unambiguously be derived from the value of $y$ (in the function given, if $y$ has the value $9, x$ could either be 3 or -3 ). 
how much time a motion takes is determined solely by the number of bodies passed. This becomes obvious, for example, in 240a10-12 (quoted above), where it is concluded that covering half the As requires half the time of covering all the Bs. But concluding from the number of spatial magnitudes passed immediately the time required entails that we understand space as the only determining factor for the time taken, and thus time as depending on space.

Prima facie this seems an unproblematic assumption, since, if $\mathrm{C} 1$ performs a motion past twice as many elements as B1 does, should not this motion go on for longer? If I walk down from Wolfson College in the North of Oxford to Blackwell's in the city centre, and you join me, but then walk not only to Blackwell's but continue on to walk further south to Christ Church Meadow, we, too, would expect you to walk for longer than I do. The further a motion goes, the more motion we have, so to speak, and hence the more time it will need. In modern conceptions too the time required for a motion is dependent on what we can call the amount of motion performed (I will need less time if I stop after a few steps than if I keep on walking) ${ }^{20}$ However, what we do if we reason like this is moving implicitly from the problematic assumption that time is dependent solely on the space covered, to the correct assumption that time is dependent on the motion performed.

But in Zeno's paradox the time is in fact not conceived of as dependent on motion, but indeed on space. This is clear from the fact that from the mere amount of bodies passed (not from the motion performed in passing them), it is inferred that B1 must have taken half the time for its enterprise of covering half the As by comparison with the time it took $\mathrm{C} 1$ to cover all the Bs. Of course, covering more space will also mean performing more motion, and this is the reason why Zeno's assumption does not strike us as wrong prima facie. However, in making the amount of time dependent on the amount of space covered, Zeno's assumption is merely in the neighbourhood, as it were, of the correct assumption that the amount of time is dependent on the amount of motion performed. To see this, let us assume that I am a slow walker, but you walk rather briskly. Then, if we both set out from Wolfson College at the same time, you might nevertheless be down at Christ Church Meadow at the same time as I arrive at Blackwell's. The fact that, in addition to the University Parks and the Natural History Museum, which we both pass, you also pass the Bodleian Library and Oriel College - and so you cover more space - does not mean that it necessarily takes you a longer time. But it would necessarily take you a longer time, if we were to assume that the amount of time needed is always dependent solely on the space covered. And this assumption is what we see at work in Zeno's paradox - a wrong assumption, but one that will suffice for many cases (for example, if we only considered Bs motion past the As).

It is clear that in Zeno's framework the time needed for a particular motion is

${ }^{20}$ As we will see below, the amount of motion performed is not only determined by distance and time, but also by the specific relation of the two, which we capture as speed. 
dependent solely on the distance covered, since the movement past a body cannot vary according to different speeds ${ }^{21}-$ it always has to take the same time. But, you might object, Zeno explicitly tells us to consider rows moving with the same speed, probably so as not to make the paradox even more complicated. So, we simply are not dealing with a case of idly walking Bs and briskly moving Cs. It might seem that, if we work with a set speed, then the two assumptions-that the amount of time is dependent on the amount of motion performed, and that the amount of time is dependent on the amount of distance covered-do indeed coincide. There would thus be a fixed relation between the space covered and the time required.

Yet to assume that B and C move with the same speed is not the same as making time completely dependent on space. If a body is in uniform motion then the time it takes to travel a certain distance bears a strict relation to that distance. But it is a strict correlation between two, in principle independent magnitudes. It is not a dependence relation. The strict correlation will hold as long as all the relevant circumstances of the motions considered stay the same, while a dependence relation would also hold if these circumstances changed. Even if we assume uniform speed, we nevertheless have to take time and space into account as independent magnitudes, so that the speed could in principle vary.

Now it seems as if Zeno does in fact take into account both time and space, since he mentions the distance passed-telling us that the magnitude of each row is of the same extension - as well as the time needed. However, as we saw above, lines 240a10-12 show that Zeno does not take time and space into account each on its own: if A and B are of the same extension, Zeno assumes, it has to take the same time to pass them..$^{22}$ When Zeno states that for the movement of the Bs as well as of the Cs the same time has to be assigned to the same number of elements passed, he is in fact not only assuming uniform speed for the Bs and Cs, but he is also making time a magnitude completely dependent on space. Otherwise, he could not immediately infer the time needed solely from the distance passed. It is not possible, on this conception, to ask: how much space was covered by $\mathrm{x}$ in a certain time? Rather, one unit of time is connected to the passing of one spatial unit in a fixed relation: one body is assumed to be covered in one time-unit. So it is in fact not the case that two different magnitudes, time and space, are used to determine movement.

But even if Zeno treats time as a magnitude completely dependent on space, why should this be a problem? Two bodies moving with the same speed will indeed cover the same distance in a stadium, and even in Oxford, in the same time. As long as all the relevant circumstances of the two bodies moving with the same speed stay the same, the assumption that the amount of time is dependent on the amount of motion performed and the assumption that the amount of time is dependent on the amount of space covered seem indistinguishable.

${ }^{21}$ I.e., Zeno has no way of articulating such a variation.

${ }^{22}$ This is especially clear in the first translation offered of the third premise of step III, 3 . 
The difficulties start once the circumstances change. This could be the result of one of the moving bodies speeding up. Then, the same time would be used for covering a bigger distance, so that it becomes obvious that, while time is indeed dependent on the motion performed, it is only correlated with the space covered. Zeno's paradox does not feature a body speeding up. But the very same problem arises if what is covered is itself in motion, so that we are not dealing with a stable distance being covered. And this kind of situation is the basic set-up of Zeno's paradox.

The University Parks, the Natural History Museum, the Bodleian Library, as well as Oriel College are normally pretty stable. So, if we pass them with the same speed, confusing time's dependence on motion with time's dependence on space will normally be unproblematic. However, if I am walking past a moving tour bus on the way down from Wolfson to the city centre, then the time needed to pass the bus cannot be immediately deduced from the distance passed-the size of the bus - since the time itself will vary with the speed of the thing being passed. But, according to the interpretation I am proposing, Zeno cannot take into account the speed of the thing being passed, since, were he to do so, time would no longer be treated as dependent solely on the distance passed. The presence of the resting As in Zeno's paradox lulls us into thinking that time is simply dependent on space, since if Bs' motion past the resting As is all that we look at, we will not get any false results. However, the fact that the Bs are also passing the Cs, which are moving in the opposite direction, reveals that the two assumptions that seem to be equivalent in the resting case in fact come apart.

It is in these cases, where a fixed connection between time and space is not sufficient to account for the motion performed, that we encounter an apparent paradox. In contrast to the motion past a resting magnitude, where treating time solely as dependent on space does not emerge as a mistake, the moving row scenario makes it obvious that motion has to be understood as a relation between time and space in which both relata can change independently of each other. In this case, where the thing to be passed itself moves towards the thing passing, the speeds of both add up. The extension of the thing passed will be passed more quickly than if the thing passed had been resting, because the distance to be covered will be lessened by the movement of the thing passing in combination with that of the thing passed. Hence, if both passing bodies share the same speed, the passage will occur twice as fast as if the thing passed were at rest.

The paradox thus arises because the time a certain motion takes is seen as dependent merely on the distance travelled, the passing of each body requires one time-unit. Thus if B passes 2 As and 4 Cs simultaneously, half the time required seems to be double the time required.

\section{Relativistic and Atomist Troubles}

Let us now come back to the three main positions in the secondary literature 
mentioned in the beginning and see what they make of our passage. ${ }^{23}$

(a) The first group maintains that no clear and interesting paradox can be derived from this text. Given this diagnosis, they dismiss it as worthless - a bad press that goes back over two millennia, to students and commentators of Aristotle. Eudemus, one of Aristotle's pupils, called this paradox 'most silly' as the fallacy is allegedly 'obvious'; and Simplicius (1895, 1019,32-1020,2) follows this assessment in his commentary on Aristotle's Physics .

(b) The diagnosis of the second group is that Zeno fails to take into account some essential relativity of motion. They do not dismiss Zeno's paradox outright, but allow that by committing this mistake, Zeno makes us implicitly aware of the relativity of motion. I will discuss Barnes here as giving the most elaborate and best known account of this group. ${ }^{24}$ The specific relativity that Barnes understands Zeno as failing to notice is that motion is relative in the sense that $a$

${ }^{23}$ An interpretation not fitting any of the three main groups is the one by Ferber 1995. He argues that the paradox rests on the mathematically (but not physically) accurate fact that $t / 2$ consists of as many points of time as $t$, since both consist of infinitely many; thus $t / 2$ equals $t$. In this way he attempts to reconstruct it as a view that is indeed philosophically interesting. However, I do not see anything in the text that allows us to ascribe this insight of the 19th century to Zeno. Ferber himself does not think there is philological evidence in the text handed down to us as the fourth paradox for his reading (cf. p. 118); and I do not think that his references to passages in other authors, like Anaxagoras' fragment 3 and On Indivisible lines 968b5-13, can indeed show that (a) this thought was already known in the fifth century BCE and that (b) our paradox may have been known to others as a paradox of infinity. (a) For example, the passage from On Indivisible lines argues that if the unit of measurement is divisible, then also parts of this unit of measurement have to be a unit of measurement, since they are commensurable with the original unit of measurement, but then half is equal to double. Ferber assumes that half the unit of measurement is equal to double, because both contain infinitely many parts. However, the text does not mention infinitely many parts. Rather, it is the commensurability of the new measure with the original measure that leads to the paradoxical result according to the text, since in order to be commensurable things have to be measurable by the same measure, and so for a part of half the size of the original unit of measurement to be commensurable with the whole unit of measurement it also needs to be measurable by the whole-thus the half is equal to the double. (b) Even if this particular knowledge about infinity had already been available at Zeno's time, what would make us believe that Zeno's paradox refers to it? Ferber refers to Anaxagoras, fragment 3 , which claims that there is always something smaller with respect to what is small and always something larger with respect to what is large, so that the small is equal to the large and everything is both large and small. One may connect this fragment of Anaxagoras with Zeno's fragment 1, that if there is a plurality of things they have to be both small and large, as Kirk and Raven 1957 do. But it seems a stretch to understand 'double the time is half the time' as a concrete example for Anaxagoras' claim, as Ferber does.

${ }^{24}$ Also Hopkins 2006, 22n40 can be counted in this group, since he thinks that the paradox 'questions the very idea of motion itself by pointing out that our idea of motion depends on identifying objects in relation'. Kirk, Raven, and Schofield 1983, 276 refer to a 'relative theory' according to which 'the distance a body moves is simply a function of its position relative to other bodies' so that there does not seem to be an absolute basis for ascribing motion to a body at all. But they do not think that such a relative theory would make us abandon our idea about the measurement of movement, which they seem to think is what actually leads us into a paradox: "if a body moves past $\mathrm{n}$ bodies of size $\mathrm{m}$, it moves a distance of $\mathrm{mn}$ units; simple arithmetic will then show that moving mn units will take half the time of moving $2 \mathrm{mn}$ units at the same speed'. Simple arithmetic will, of course, show this only, if we assume time to be completely dependent on the distance covered. 
always moves in relation to some $b$. Thus, ' $a$ moves' has to be understood 'as elliptical for " $a$ moves vis-à-vis $b$ ", (Barnes 1982, 293). Speaking more extensively, Barnes thinks that the way motion is linked to time and space is 'mirrored in these two implications: (1) If $a$ moves past a sequence of $n F$ s and each $F$ is $k$ units long, then $a$ moves $n k$ units' and (2) 'If $a$ moves for a period of $T$ units at a constant speed of $j$ u.p.u., and covers $m$ units in that time, then $T=m / j$ units' (291). ${ }^{25}$ In the case of Zeno's set-up, however, these two implications seem to lead to $T=1 \mathrm{k} / \mathrm{j}$ for the passage of B 1 past a single A and to $T=2 \mathrm{k} / \mathrm{j}$ for the simultaneous passage of B1 past two Cs. The reason we get entangled in the paradox is, according to Barnes, that these two implications do not factor in that motion is relative in the sense specified above. If we take this relativity into account, then (1) has to be transformed into $\left(1^{*}\right)$ in order to keep its essence while avoiding the paradoxical consequences: ' $\left(1^{*}\right)$ If $a$ moves past a sequence of $n F$ s and each $F$ is $k$ units long, then relative to those $F$ s $a$ moves $n k$ units'. Thus Barnes thinks we get " $a$ moves $n$ units relative to $b$ " and " $a$ moves $m$ units relative to $c$ "' (p. 293), which is without any contradictions.

Prima facie, Barnes's reconstruction of the paradox with the help of the two conditionals (1) and (2) seems plausible. The first seems to give us the space covered, the second the time taken by the motion in question given a certain speed. The evaluation Barnes derives from the diagnosis that Zeno fails to notice the relativity of motion is that it is an understandable failure at Zeno's time and one we are vulnerable to ourselves in unthinking moments. ${ }^{26}$ However, there are some problems with Barnes's account such that in fact it neither gives us a correct diagnosis of Zeno's paradox, nor an account of how successfully to avoid the paradox.

Pointing out the relativity of motion in Barnes's sense is not enough in order to block the paradox. For Zeno shows clear awareness of the relativity Barnes is talking about, that something moves not simpliciter but with respect to something else. At the beginning of the paradox Zeno makes explicit a shift in perspectives: he tells us that we are dealing with $\mathrm{Cs}$ movement past the Bs, and Bs movement past the As; we are not just told that the Bs move or the Cs move. Further, Zeno takes into account that the distance passed is relative to the thing passed; B is moving four units in relation to $\mathrm{C}$ as well as two units in relation to A. So unless we are reading Zeno uncharitably and claim that he fails to consider this relativ-

25 Barnes does not spell out the second implication and the result $T=m / j$ any further, but he seems to understand by ' $T$ ' units of time, by ' $m$ ' units of space, and by 'u.p.u' units per units, i.e., in our case spatial units per temporal units. So B1 moves for a period of 2 temporal units at a constant speed of 1 u.p.u. and covers 2 spatial units (the two As).

26 The account of the paradox in Furley 1967 also boils down to attributing a mere oversight to Zeno, albeit an oversight based on a mistaken assumption of transitivity, rather than on lack of understanding of some relativity of motion: if $\mathrm{B}$ is opposite $\mathrm{A}$ for one unit of time and $\mathrm{C}$ is opposite $\mathrm{A}$ for one unit of time then, by transitivity, $\mathrm{B}$ must be opposite $\mathrm{C}$ for one unit of time. According to this interpretation, Zeno simply overlooks the fact that because the Bs and the Cs approach the As from different sides, it is only when B1 has already passed half of the As that it will actually start to be opposite $\mathrm{C} 1$ as well - so the transitivity assumed does not hold. 
ity that he is aware of, this all indicates that the relativity of the distance travelled is taken into account by Zeno. Hence, making it explicit by changing (1) to $\left(1^{*}\right)$ does not help to avoid the paradox.

Within the Zenonian framework, also $\left(1^{*}\right)$ will entangle us in the problem that $\mathrm{B} 1$ moves 2 times 1 unit relative to $\mathrm{A}$ and 4 times 1 unit relative to $\mathrm{C}$ during the same time, even though each $\mathrm{A}$ and $\mathrm{C}$ are of the same extension and B does not change its speed. Making the distance travelled relative to the row passed (as Barnes tries to do with $(1 *)$ ), rather than to the motion of the row passed, does not explain why passing units of the same extension, and with the same speed, gives us different distances passed within the same time. What is important to understand (and thus ultimately also to avoid) the paradox is not that everything moves relative to something else, but rather that while the time of a motion is correlated to the distance covered, it cannot simply be derived from this distance. Putting it in the terms of Barnes's account, what has to be changed is the assumption we find in (2), since it does not allow us to take into account that - even if B is moving with constant speed-passing distance $m$ might nevertheless take different times. Indeed if we simply insert the result we get from $\left(1^{*}\right)$ in $(2)$ as it stands, then we derive two different times for the same motion: $T$ is $m / j$ on the one hand and $n / j$ on the other. So we are back to Zeno's conclusion that double the time is half the time: whereas it takes B the same time to pass two As and four $\mathrm{Cs}$, it should, according to Barnes's ( $1 *$ ) and (2), take $m / j$ units for passing the As and $n / j$ for passing the Cs.

(c) Finally, the third main group of interpreters diagnoses this paradox as a challenge to atomism, ${ }^{27}$ and understands it to be of genuine philosophical substance. Most often, this group reconstructs the paradox as directed against time atomism, but sometimes time and space atomism are combined as alleged points of attack.

In its original version, in Tannery 1877, this account included the assumption that Zeno's paradox was directed at Pythagorean atomism..$^{28}$ Once the assumption of Pythagorean atomism was no longer widely shared, several scholars, especially in Britain, rejected this interpretation, regarding it as a historical mistake. ${ }^{29}$ If the Pythagoreans did not posit atoms, there were no atomists around at the time that Zeno developed his paradoxes and hence none to be their target.

${ }^{27}$ Atomism is broadly construed here: it includes all kinds of assumptions of indivisibles, so, e.g., indivisible units of matter (allegedly to be found in Empedocles to which Zeno's onkoi refer, according to Gaye 1908, 114f.), as well as the mere treatment of magnitudes as discrete and indivisible units (according to Mansfeld 1982, 18 body, motion, time, and space do not consist of indivisible minima but once they are divided into sections, these sections are treated as if they could not be broken up in Zeno's paradox, cf. also n29). I group all these kinds of indivisibles under the term 'atoms' in my discussion, since this is the term most often used in reconstructions of the paradox in this vein.

28 Tannery 1877, 266. Tannery assumed Pythagorean atoms to be point-like, but interpretations of the paradox with extended atoms also came into fashion on the basis of his interpretation.

${ }^{29}$ In the English speaking world the idea that the Pythagoreans assumed atoms was prominently defended by Cornford. For the dismissal of this idea see especially Booth 1957a; Furley 1967, 44-56; Owen 1957-8; and Barnes 1982, 291. 
Nevertheless, the atomist interpretation remains the only philosophically substantive reading in the secondary literature to date that attempts to give an account of the text as handed down to us and outside of Britain it still enjoys a lot of popularity. ${ }^{30}$ Accordingly, it is worth revisiting this position briefly, especially since it is normally rejected due to historical inaccuracy, while I want to show that it can be rejected also on purely philosophical grounds.

I will concentrate on the most widespread branch of atomist interpretations, which presumes that the reason we get entangled in a paradox is the assumption of indivisible time atoms. ${ }^{31}$ The diagnosis these atomist interpretations give can be roughly characterized as follows: Zeno assumes that there are smallest, indivisible time atoms, and that one body (one letter element) will be passed in one indivisible time atom. But if we look at the time atom in which B1 passes A3, we find that during that same indivisible time atom, B1 has passed two elements of the $\mathrm{C}$-row, $\mathrm{C} 1$ and $\mathrm{C} 2$. According to the premise, however, the passing of each element is connected with one indivisible time atom, so that double the time - the two time atoms required to pass $\mathrm{C} 1$ and $\mathrm{C} 2$-equals half the time-the one time atom that is necessary to pass the single $\mathrm{A} 3 .^{32}$

There are three main points speaking against such atomist interpretations of the paradox: (I) There is no good evidence of atomism in the text-there is no reference whatsoever to an assumption of time atoms. ${ }^{33}$ (II) If this were a paradox against atomist assumptions, it would establish only that atomists have a problem in giving a consistent account of motion, and thus would have only a rather limited target (whether or not there were indeed already atomists around at Zeno's time). Some people, adhering to the seemingly appealing idea that the first two of Zeno's paradoxes of motion are directed against understanding time and space as a continuum, while the third and fourth are directed against time and space conceived as atomistic, might think that this reduced target does not matter, since the

${ }^{30}$ Cf., e.g., Salmon 1980 and Glazebrook 2001. The atomist interpretations were replaced in Britain by 'transitivity interpretations', of the sort we encountered with Furley, or, more widely spread, by 'relativistic interpretations' of the kind we saw in Barnes.

${ }^{31}$ Among the supporters of time atoms as prerequisite for this paradox we find, e.g., Russell 1903, 352-353, §334; Salmon 1980, 34-35; and Kirk and Raven 1957 (this was changed, however, in Kirk, Raven, and Schofield 1983).

${ }^{32}$ Space atomism assumes that the bodies passed are indivisible, so that it is impossible to be partially alongside a single body (one letter element). So one body is either opposite or not opposite another, which is problematic when B passes one atomic element $\mathrm{A}$, and thus is opposite $\mathrm{A}$, at the same time as it passes two atomic Cs. And mixed atomism assumes both, that the distance of a body cannot be only partially passed, and that no motion that would take half a time unit can be performed; cf. Ross in Aristotle 1936, 81-84; and Cornford in Aristotle 1934 ad loc.

${ }^{33}$ Space atomists might claim that the distances to be passed are bodies, onkoi, which are represented as letter elements and hence as atomistic (this is, e.g., how Gaye 1908, 111ff. understands the term onkoi). However, both Furley 1967, 73 and Booth 1957b, 193-194 deny that the term onkos indicates that the magnitudes are indivisible. The separation into letter elements may simply be designed to mark out spatial units, which we need for the paradox, without implying indivisibility, cf. below. Further, if this paradox did indeed rest on an atomist assumption, we would expect Aristotle in his fierce opposition to atomism to have pointed this out (cf. also Booth 1954b, 195). 
non-atomist alternative is accommodated by means of the first two paradoxes. However, along with Kirk, Raven, and Schofield as well as Barnes, I think this interpretative scheme does not work, as can be shown with reference to the other paradoxes of motion also. ${ }^{34}$ In any case, it seems to be clear that the paradox of the Moving Rows is stronger, if - as on my interpretation - it is not directed solely against atomism.

(III) The most important objection against any kind of atomist interpretation, however, is the fact that the paradox in no way relies on atomist assumptions. We will be caught up in the paradox whether we start from the assumption that time is structured continuously, i.e., that it can be divided ad infinitum, or that it is structured discretely, i.e., that we will reach indivisible magnitudes, atoms, after a finite number of divisions. So the crucial point of the paradox is not the assumption of atoms; and if we leave out what I pointed out above as the crucial assumption of the paradox, time's dependence on space, atomist interpretations wind up reconstructing a paradox with a different punch-line from the one handed down to us as Zeno's.

To show that the paradox is independent of any atomist assumptions, let us first assume that time is a continuum in which continuous rows move, and we will see that we get into the same paradox. In our continuous set-up there are four different sections in each row. But these sections are just marked out in order to allow for talking about different segments, just as we mark out a big table for measuring when using a small ruler. They are not naturally fixed parts; and they are further divisible as we like. ${ }^{35}$ Similarly, time can be marked out into different units for measuring purposes; but these units are in themselves further divisible ad infinitum. And the continuous $\mathrm{B}$ and $\mathrm{C}$ rows each cover one section in one unit of time. So we can assume that the segment B1 from the continuous B-row passes the segment A3 in one time unit. But at the same time, B1 will pass two

${ }^{34}$ Cf. Kirk, Raven, and Schofield 1983, 265 who remark that 'Such schemes are undoubtedly attractive, but none has any ancient authority, nor have they withstood critical scrutiny very well', and Barnes 1982, 285. Another such scheme claims that each of the four paradoxes argues against one of the four possibilities concerning the divisibility of time and space: space is infinitely divisible and time is finitely divisible; space is finitely divisible and time is infinitely divisible; both are infinitely divisible; both are finitely divisible, cf. Glazebrook 2001, 194. Like the self-declared atomistic interpretations, also this scheme argues that the moving rows paradox assumes time and space to be only divisible up to a certain point, which is not what creates the paradox, as I show in the following. (Glazebrook thinks that this scheme is a strategy to attack the Pythagorean fusion of the mathematical and the physical by showing that 'whatever application it [a mathematical concept, like infinity] is given to physical entities results in absurdity'. It is unclear, however, why finite divisibility, as one horn of the dilemma this scheme works with, should be a mathematization of nature and not something that is indeed encountered in the natural world). Cf. also Brochard 1954 who sees a couple of such schemes at work.

${ }^{35}$ Cf. also Wagner in Aristoteles 1995, 639. It is true that the use of the plural onkoi and of $\tau \grave{\alpha}$ $\mathrm{AA}, \tau \grave{\alpha} \mathrm{BB}$, and $\tau \grave{\alpha} \Gamma \Gamma$ clearly refers to more than one distinct body and thus would not be used if each AA, BB, and $Г \Gamma$ were one continuous body. But since we will see that we get into the same paradox on the assumption of one continuous body with different sections marked out, the assumption of a plurality of bodies turns out not to be necessary for the paradox. 
segments of the C-row, and thus it should need two (divisible) units of time. Time being a continuum, there would be no problem in dividing the fixed time unit into smaller portions, for instance into two halves. ${ }^{36}$ Hence, indivisibility cannot be what leads us into this paradox. Rather, the problem is that we started with the assumption that one time unit is connected with passing one spatial segment, and now, while B1 is passing exactly one A segment in this time unit, it is also passing two $\mathrm{C}$ segments at the same time. Thus double the time-two units of time for passing two $\mathrm{C}$ segments - equals half the time-one time-unit for passing one A segment.

If we assume, on the other hand, that time is atomistic in character without fixedly connecting one time atom with the passing of one element we get the following scenario: B1 passes A3 in one time atom, but at the same time it also passes two Cs. So either (1) B1 passes one $\mathrm{C}$ in half a time atom although a time atom is per definition not divisible; or, as some scholars assume as an alternative (e.g., Salmon 1980), (2) B1 passes two Cs in one time atom in such a way that it never actually is opposite the first C. ${ }^{37}$

Whichever of the two interpretations (1) or (2) we may choose, the paradox the atomist interpretation leads us into is a different paradox from the one Aristotle is giving an account of. If we choose the first interpretation, we will face the paradox that, although we are assuming a time unit to be indivisible, we have to think of it as being divisible, for two different elements are covered in it. ${ }^{38}$ The paradox we are dealing with, however, is not that indivisible minima have to be divisible, but rather that half the time is double the time. ${ }^{39}$

Opting for the second interpretation, we either get the paradox that, although $\mathrm{B} 1$ somehow passes $\mathrm{C} 1$ as well as $\mathrm{C} 2$, there is no point when it is opposite $\mathrm{C} 1$; so B1 is moving past C1 without ever passing it (cf. Salmon 1980, 35). This paradox, too, is not the one we are looking for, but rather the puzzle of how a thing could pass something without ever being opposite it. Or we could, as Sorabji 1983, 331 suggests, take this interpretation to give an adequate description of

${ }^{36}$ And since space is not atomistic, partial overlap is possible.

${ }^{37}$ Assuming space atomism, we find that B1 passes A3 in one time-unit, but simultaneously it also passes two Cs, so B1 should pass the first $C$ when it has covered half of A3. Thus either (1) B1 passes one $\mathrm{C}$ when it has passed half $\mathrm{A} 3$ even though $\mathrm{A} 3$ is not divisible and there cannot be a partial overlap, or (2) in one time-unit B1 passes two Cs and A3 in such a way that the two Cs are never partly opposite the one indivisible A in their passing. Mixed atomism will combine time and space atomism (Brochard 1954, 8 who assumes mixed atomism, essentially reconstructs the paradox as one of time atomism, and then adds a sentence translating it into a problem of space atomism).

${ }^{38}$ Cf. Russell, p. 352: 'hence the instant is divisible, contra hyp.'

${ }^{39}$ Brochard 1954, 9 simply translates the paradoxical result, that the indivisible instant is divisible into two equal parts, into the claim that the double is the same ('Dire que l'instant est divisé en deux parties égales, c'est, dans l'hypothèse, dire qu'il est le double de lui-même'). But he does nothing to explain why Zeno should have claimed to show that double the time is half the time, when what he really does is showing that what is supposed to be indivisible needs to be divisible. That the atomistic paradox is the one also Brochard ultimately takes to be the heart of the matter, can also be seen from his final account of his interpretation of the paradox on p. 11, which claims it to show that in a motion the supposedly indivisible instant can be divided. 
motion within an atomistic framework ${ }^{40}-\mathrm{B} 1$ 'passes these two [Cs] all in one go, by disappearing from where it was and reappearing futher on'. But then we get no paradox at all from Zeno's fourth so-called paradox. ${ }^{41}$

Atomist reconstructions of the paradox thus have a different punch line from the one the paradox as handed down to us offers: they demonstrate either that if we start by supposing some minimal temporal periods to be indivisible, we end up after all having to conceive of time as divisible if we are to accommodate motion, or that, when passing some supposedly indivisible minimal extension, we are never actually opposite it, so that motion occurs in jerks. Further, if one wanted to get an atomist paradox much less machinery would suffice. All you would need to do is what Aristotle does in Physics vi, namely, to ask 'if A passes B in one time atom, what happens if A moves twice as fast?' Why, then, should Zeno build up this rather elaborate scene in the paradox (which, judging from the manuscript tradition, confused at least some of the scribes) in order to make a point that he could have made without any large and confusing machinery?

\section{Conclusion}

Summing up, we can say that none of the three main positions in the scholarly literature gives a satisfying analysis of the paradox. The first simply thinks that there is no interesting paradox. The second claims some relativity to be overlooked by Zeno, but can neither reconstruct the paradox as it is reported to us nor show how it can be avoided. And the atomist interpretation, the third position, is not needed to generate the paradox, nor is it mentioned, and it would get us a different paradox from the one handed down to us as Zeno's.

Hence, what leads into the paradox is not the premise that there are indivisible time atoms, nor that something is always moving relative to something else. Rather, the paradox arises as a result of the implicit assumption that time is regarded as a magnitude completely dependent on space, so that one time unitwhether indivisible or not-is always connected to the passing of one space unit. Understood in the way I have proposed, the paradox of the Moving Rows works

${ }^{40}$ Sorabji himself thinks the problem with the paradox is actually not atomism but the relativity of motion.

${ }^{41}$ There is one other way of reconstructing the paradox with the help of space atomism, which Stephen Menn (personal communication) seems to hold. We start out with Furley's transitivity assumption - if B is opposite $\mathrm{A}$ for one unit of time and $\mathrm{C}$ is opposite $\mathrm{A}$ for one unit of time then, by transitivity, B must be opposite $\mathrm{C}$ for one unit of time. Now we also assume space atomism, so that the transitivity assumption is not merely an oversight by Zeno, but based on space atomism. On this account each body passed is indivisible, so being opposite the As cannot be partial. Accordingly, it cannot be the case that $\mathrm{B} 1$ is opposite $\mathrm{C} 1$ when it has passed half of $\mathrm{A} 3$. However, if this interpretation wants to arrive at the paradox that half the time is double the time (and not merely that space is divisible, contra hyp., or that $\mathrm{B} 1$ is never really opposite $\mathrm{C} 1$ ), then it also has to make the assumption I pointed out above as being responsible for the paradox, namely, that time is treated as a magnitude solely dependent on space. Only thus will the time during which the Bs and the Cs are opposite the As seem to be half the time during which the Bs and Cs are opposite each other. But if we make this 
in the way we expect a Zenonian paradox to work: there are no simple mistakes in the set up of the paradox; it exploits everyday intuitions (that are, in fact, only of limited reliability); and it shows that they get us in trouble when we try to give an account of motion. Finally, the paradox addresses itself to all natural philosophers, and not only to those that are committed to atomism.

My account also explains why the paradox requires such an elaborate and complicated set-up. Admittedly, the problem Zeno raises could also have been shown, in a less complicated way, with the help of an example involving change of speed; if, for instance, the thing that is moving were to accelerate. ${ }^{42}$ But change of speed is not a concept that was sufficiently analysed in Zeno's time, or indeed at any time in the ancient period (it seems to be a change of a change, which, for example, Aristotle's Physics explicitly claims to be problematic). ${ }^{43}$ Thus, in order to get the same result as one gets with an example involving acceleration - that the relation between time and space does not stay constant-Zeno had to devise a rather complicated scenario, the one I have given an account of here.

The main assumption that leads into Zeno's paradox is that, with respect to a certain motion, time and space are dependent on each other. This assumption seems to hold when we consider the case of one body moving with constant speed past some unmoving extension, as when, for example, one train passes the stationary platform of the train station south of Elea. However, the correct assumption that is in the neighbourhood of this supposition (and that allows us to avoid the paradox) is that, with respect to this motion, time and space are closely correlated. In fact, they are so closely correlated that if all other parameters regarding a motion stay the same, we can treat them as if they were dependent on each other. This is the reason why Zeno's shortcut-making time directly dependent on space-may seem unproblematic. But time and space are in fact not dependent on each other, as becomes clear when one parameter changes, when, for example, the extension being passed is itself moving, as when one moving train passes another moving train.

Accordingly, this paradox engenders the task of understanding time and space as two independent magnitudes, which are nevertheless correlated via motion. And motion has to be understood as a variable relation of time and space. One reason why Aristotle - the most important person dealing with this paradox in ancient times-dismisses this paradox quickly without showing us how to solve it (which he does in the case of the other motion paradoxes) seems to be the fact that he himself does not manage to conceptualize motion as such a variable relation of time and space. In his comparison of motions with different speed in Physics vi the time of a motion is indeed treated as correlated with but not dependent on the distance covered (this suggests that Aristotle is aware that time is not

assumption, we do not need any form of atomism to generate the paradox.

42 Or, for a reverse Moving Rows paradox, if the thing moving decelerates.

${ }^{43}$ Cf. Physics 225b13-21 and also Bostock 1991, 205. I have tried to show in Sattler in preparation that even the concept of speed is a problematic one in the writers before Aristotle and indeed in 
simply dependent on the distance covered). However, in his explicit account of motion it is only time that he conceptualizes as determining motion. Space (or another second magnitude in the case of changes other than change of place) is quietly dropped. ${ }^{44}$ While a full analysis of motion would also have answered the paradox of the Moving Rows, it seems that Aristotle himself cannot conceptualize motion in such a way that time and space are correlated without being dependent on each other.

In this way this paradox tells us also something about the difficulty of conceptualizing motion and speed-difficulties we are usually not aware of. The history of natural philosophy shows that it would take roughly another 2000 years to give an adequate account of motion as a relation of time and space in which time and space are two independent magnitudes. It is only once such an account is given that the two assumptions, which are often not distinguished-time is dependent on the space covered and time is dependent on the motion performed - can actually be distinguished; a distinction that is necessary to avoid getting entangled in Zeno's paradox. ${ }^{45}$

Department of Philosophy

University of St. Andrews

Saint Andrews, Fife KY16 9AJ, United Kingdom

\section{BIBLIOGRAPHY}

Aristotle. 1934. Physics, vol. 2. Philip H. Wicksteed and Francis M. Cornford trans. Cambridge: Harvard University Press.

Aristotle. 1936. Physics. revised Greek text with introduction and commentary by William David Ross. Oxford: Clarendon Press.

Aristoteles. 1995. Physikvorlesung. 5th edn. Hans Wagner trans. Berlin: Akademieverlag.

Bostock, David. 1991. 'Aristotle on Continuity in Physics VI' 179-212 in Judson ed. 1991.

Barnes, Jonathan. 1982. The Presocratic Philosophers. revised edn. London and New York: Routledge.

Booth, N.B. 1957a. 'Were Zeno's Arguments Directed against the Pythagoreans?' Phronesis 2: 90103.

Booth, N.B. 1957b. 'Zeno's Paradoxes' Journal of Hellenic Studies 77: 187-201.

Brochard, Victor. 1954. 'Les argument de Zénon d'Élée contre le mouvement' Études de philosophie ancienne et de philosophie moderne. Paris: J. Vrin.

Ferber, Rafael. 1995. Zenons Paradoxien der Bewegung und die Struktur von Raum und Zeit. 2nd edn. [1st edn. 1981]. Stuttgart: Franz Steiner Verlag.

Furley, David. 1967. Two Studies in the Greek Atomists. Princeton: Princeton University Press.

Gaye, R.K. 1908. 'On Aristotle Physics Z IX 239b 33-240a 18 (Zeno's Fourth Argument against Motion)' Journal of Philology 31: 95-116.

Glazebrook, Trish. 2001. 'Zeno against Mathematical Physics' Journal of the History of Ideas 62:

Aristotle's Physics.

${ }^{44}$ Cf. esp. Physics iv and vi. I attempt to explain why Aristotle could not conceptualize motion as a correlation of time and space in Sattler in preparation.

45 The first version of this article owed a lot to Ulrich Bergmann's inspiration and criticism. I want to thank the ancient philosophy audience of King's College London, where I gave a later version in their Old Chestnuts series, Sarah Broadie, David Sansone, Zoltan Szabo, and an anonymous referee for Ancient Philosophy for their suggestions. Special thanks to Verity Harte and Michael 
193-210.

Hopkins, Phil. 2006. 'Zeno's Boêtheia Tôi Logôi: Thought Problems about Problems for Thought' Epoché 11: 1-25.

Judson, Lindsay ed. 1991. Aristotle's Physics: A Collection of Essays. Oxford: Clarendon Press.

Kirk, Geoffrey S., John E. Raven. 1957. The Presocratic Philosophers, a critical history with a selection of texts. Cambridge: Cambridge University Press.

Kirk, Geoffrey S., John E. Raven, and Malcolm Schofield. 1983. The Presocratic Philosophers, a critical history with a selection of texts. Cambridge: Cambridge University Press.

Knorr, Wilbur. 1983.'Zeno's Paradoxes Still in Motion' (Essay review on Rafael Ferber's Zenons Paradoxien der Bewegung und die Struktur von Raum und Zeit) Ancient Philosophy 3: 55-66.

Mansfeld, Jaap. 1982. 'Digging Up a Paradox: A Philological Note on Zeno's Stadium' Rheinisches Museum für Philologie 125: 1-24.

Miller, Stephen. 2004. Ancient Greek Athletics. New Haven: Yale University Press.

Owen, G.E.L. 1957-8. 'Zeno and the Mathematicians' Proceedings of the Aristotelian Society 58: 199-222.

Russell, Bertrand. 1903. Principles of Mathematics. Cambridge : Cambridge University Press.

Salmon, Wesley. 1980. Space, Time and Motion. A Philosophical Introduction. 2nd revised edn. Minneapolis: University of Minnesota Press.

Sattler, Barbara. in preparation. Natural Philosophy in Ancient Greece-The logical, methodological, and mathematical foundations of motion. Book manuscript.

Sattler, Barbara. under review. 'Reconstructing Zeno's Fourth Paradox of Motion'.

Simplicius. 1895. In Aristotelis Physicorum. Libros Quattuor Posteriores Commentaria in Commentaria in Aristotelem Graeca. Edita consilio et auctoritate Academiae litterarum regiae borussicae. Vol. X. Hermann Diels ed. Berlin: Verlag Georg Reimer.

Sorabji, Richard. 1983. Time, Creation and the Continuum, Theories in Antiquity and the early Middle Ages. London: Duckworth.

Tannery, Paul. 1877. Pour L'Histoire de La Science Hellène. Paris : F. Alcan.

Vlastos, Gregory. 1966. ‘A Note on Zeno's Arrow' Phronesis 11: 3-18. 